\title{
Extraction of Discriminative Functional MRI Activation Patterns and an Application to Alzheimer's Disease
}

\author{
Despina Kontos ${ }^{1}$, Vasileios Megalooikonomou ${ }^{1}$, Dragoljub Pokrajac ${ }^{2}$, \\ Alexandar Lazarevic ${ }^{3}$, Zoran Obradovic ${ }^{1}$, Orest B. Boyko ${ }^{4}$, James Ford ${ }^{5}$, \\ Filia Makedon ${ }^{5}$, and Andrew J. Saykin ${ }^{6}$ \\ ${ }^{1}$ Department of Computer and Information Sciences, Temple University, Philadelphia, PA \\ \{dkontos, vasilis\} atemple.edu, zoranaist.temple.edu \\ ${ }^{2}$ Department of Computer Science, Delaware State University, Dover, DE \\ dragoljub.pokrajac@verizon.net \\ ${ }^{3}$ Department of Computer Science, University of Minnesota, Minneapolis, MN \\ alex@cs.umn . edu \\ ${ }^{4}$ Department of Diagnostic Imaging, Temple University, Philadelphia, PA \\ oboyko@temple.edu \\ ${ }^{5}$ Department of Computer Science, Dartmouth College, Hanover, NH \\ jford@cs.dartmouth. edu, makedon@cs.dartmouth. edu \\ ${ }^{6}$ Brain Imaging Laboratory, Departments of Psychiatry \& Radiology, Dartmouth Medical \\ School, Lebanon, NH \\ saykin@dartmouth.edu
}

\begin{abstract}
We propose a novel Dynamic Recursive Partitioning approach for discovering discriminative patterns of functional MRI activation. The goal is to efficiently identify spatial regions that are associated with non-spatial variables through adaptive recursive partitioning of the $3 \mathrm{D}$ space into a number of hyperrectangles utilizing statistical tests. As a case study, we analyze fMRI datasets obtained from a study that explores neuroanatomical correlates of semantic processing in Alzheimer's disease. We seek to discover brain activation areas that discriminate controls from patients. We evaluate the results by presenting classification experiments that utilize information extracted from these regions. The discovered areas elucidated large hemispheric and lobar differences being consistent with prior findings. The overall classification accuracy based on activation patterns in these areas exceeded $90 \%$. The proposed approach being general enough has great potential for elucidating structure-function relationships and can be valuable to human brain mapping.
\end{abstract}

\section{Introduction}

The detection of relationships between human brain structures and brain functions (i.e., human brain mapping) has been recognized as one of the main goals of the Human Brain Project [1]. Several approaches have been used in this problem domain [2] One of the approaches used in functional brain mapping is to seek associations between brain activation patterns and tasks performed. A current obstacle in this type of analysis is the lack of methods to automatically classify such patterns (i.e., activation regions) and quantitatively measure levels of their similarity. In this paper, we focus 
on analyzing patterns of brain activity obtained using functional Magnetic Resonance Imaging (fMRI).

One of the most common approaches currently in use, statistical parametric mapping (SPM) [3] analyzes each voxel's changes independently of the others and builds a corresponding map of statistical values. The significance of each voxel is ascertained statistically by means of Student's t-test, F-test, correlation coefficient, or other univariate statistical parametric tests. The multiple comparison problem, which occurs when computing a statistic for many pairwise tests (introducing significant computational overhead), is usually handled by estimating corrected p-values for clusters.

Another approach to the problem is to model (estimate) the underlying distributions of the distinct classes (controls vs. patients) [4-5], utilizing parametric, nonparametric or semi-parametric techniques. EM and k-means algorithms [6] have been used in order to estimate the distribution densities. Statistical distance based methods are often used for distinguishing among distributions. The Mahalanobis distance [7] and the Kullback-Leibler divergence [6] are most often employed. The main problem of these techniques is that real data are not accurately modeled using a simple mixture of Gaussian components, since they correspond to highly non-uniform distributions.

In the proposed approach, we use an adaptive recursive partitioning approach on the $3 \mathrm{D}$ domain to discover highly informative $3 \mathrm{D}$ sub-regions with respect to the development of a disease. The method operates on brain activation maps generated by SPM when analyzing the subjects independently (post-analysis of activation maps has been shown to be very useful [8]). More specifically, we utilize Dynamic Recursive Partitioning (DRP) initially presented in [9] for the analysis of binary artificial and realistic data. Some initial attempts to apply the technique on brain images have been reported in [10]. Here, we present a detailed description of how we extended DRP in order to be applicable to real 3D functional activity data. We also present the results of a comprehensive study on a collection of datasets obtained from a series of semantic decision tasks designed to explore neuroanatomical correlates in Alzheimer's disease (AD) [11]. These results clearly demonstrate the ability of DRP to identify discriminative spatial patterns arising from functional imaging information, assisting in medical decision making. We also investigate the case of developing a classification model based on neural networks that utilizes information extracted from the subregions indicated by DRP to provide prediction and diagnosis.

\section{Methodology}

We seek to discover highly discriminative regions with respect to class membership (controls vs. patients). In the discussion that follows we present the method for a twoclass problem although it can be easily extended to more than two classes. In order to evaluate the method we also seek to construct features (attributes) that can be used to develop and train a classification model for prediction and medical diagnosis.

The method is applied on activation maps that are the output of SPM (operating on individual subjects independently). SPM creates 3D activation maps of contrast and statistical significance values for pairs of conditions. The proposed algorithm treats the initial 3D volume of activation maps as a hyper rectangle and searches for infor- 
Given: Oct-tree $T$ corresponding to the spatial domain D; Two sets $S_{Y}=\left\{S_{l, Y}, \ldots S_{n l, Y}\right\}, S_{N}=$ $\left\{S_{I, N}, \ldots S_{n 2, N}\right\}$ containing region data for samples belonging to classes $\mathrm{Y}$ and $\mathrm{N}$ respectively.

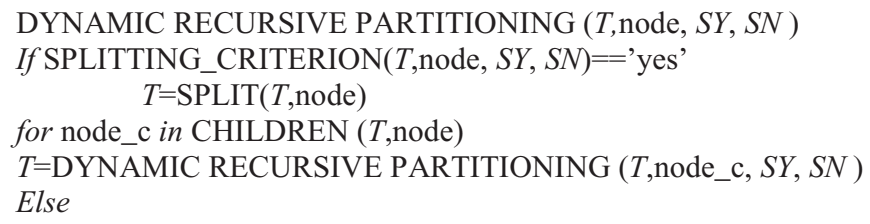

Fig. 1. The outline of the DRP algorithm in pseudocode

mative regions by partitioning the space into sub-regions (cuboids), in an adaptive way. We use the mean $\mathrm{V}_{\text {mean }}$ of all voxel values belonging to the cuboid under consideration as a measurement of activation/deactivation level. This measurement is treated as a candidate feature (attribute) for the corresponding sub-region. The adaptive partitioning of the 3D space continues in the following way: A hyper-rectangle is partitioned only if the corresponding attribute does not have a sufficient discriminative power to determine the class of samples. This is determined by the use of statistical tests, where a statistical significance threshold is employed (e.g. p-value $<0.001$ ) as a stopping criterion for splitting. The procedure progresses recursively until all remaining sub-regions are discriminative or a sub-region becomes so small that it cannot be further partitioned. For this reason, the maximum number of partitioning steps (depth) that the partitioning can go through is also predefined. For the implementation of this procedure, efficient data representation and manipulation is accomplished using augmented oct-trees [12] and a dynamic array [13] to store pointers to the leaf nodes. If the splitting criterion is satisfied, the spatial sub-domain (or cuboid) corresponding to the node of the oct-tree is partitioned into 8 smaller sub-domains. The corresponding tree node becomes the parent of eight children nodes, each representing a new sub-domain. The new measurements $\mathrm{V}_{\text {mean }}$ corresponding to the children nodes become the new candidate attributes. Figure 1 shows the outline of the DRP algorithm.

As described above, the adaptive partitioning of the 3D space is guided by a statistics-based stopping criterion. Several statistical tests can be applied for this purpose. For example, the Pearson correlation coefficient [14] between the class label (considered as a binary numeric value) and the attribute value for each sample $\left(\mathrm{V}_{\text {mean }}\right)$ could be computed and an attribute considered significant if the correlation coefficient is larger than the pre-determined threshold. Another criterion is based on discretization of the candidate attribute and evaluation of the class/attribute contingency matrix using statistical tests (chi-square or the Fisher exact test [15]) with pre-determined maximal type I errors. A suitable value for the discretization threshold can be set adhoc or by using discretization techniques that maximize class/attribute mutual information [16]. Finally, the significance of a candidate attribute can be assessed by deciding whether the distributions of attribute values corresponding to the classes differ substantially using parametric (e.g. t-test [14]) or non-parametric tests (e.g. Wilcoxon rank sum [17]). 
The proposed method effectively reduces the number of times a statistical test is applied due to the adaptive approach that is used. This is because the statistical tests are applied selectively on groups of voxels (cuboids), focusing only on certain potentially discriminative sub-regions. This is in contrast to the traditional voxel-wise application of statistical tests, such as in SPM [3], were repeated statistical tests on a voxel-wise basis introduce the multiple comparison problem (see Section 1).

\section{Experimental Evaluation}

\subsection{The Dataset and Preprocessing}

Our dataset consisted of 3D activation maps of 9 Alzheimer's disease patients and 9 elderly controls. The brain activation data were collected during a series of cognitive tests [11]. These tasks were selected to differentially probe semantic knowledge of categorical, functional, and phonological congruence between word pairs: (a) Category exemplar (catx): identify word pairs with correct category exemplar relationships from among incorrect ones, (b) Category function (catf): identify word pairs with correct category function relationships from among incorrect ones, (c) Nonsense pairs (nonpr): listen to nonsense pseudo-word pairs and decide if they are the same or different, and (d) Episodic recognition memory task (imprec): identify formerly heard words and pseudowords encountered in catx and catf tasks above versus new foils.

The word pairs were presented in groups of four at 7.0 second intervals, with each 28.0 second block of decision followed by a 10.5 second period of rest. Scans were conducted at 1.5 Tesla using a single shot, gradient echo, echo planar functional scan sequence $(\mathrm{TR}=3500 \mathrm{~ms}, \mathrm{TE}=40 \mathrm{~ms}$, interleaved, $\mathrm{FOV}=24 \mathrm{~cm}$, slice thickness $=6$ $\mathrm{mm}, \mathrm{NEX}=1$, flip angle $=90$ ) on a General Electric Signa scanner with a multi-axial local gradient head coil system (Medical Advances, Inc., Milwaukee, WI). Scans consisted of 20-23 contiguous sagittal slices in a 64x64 matrix with in-plane resolution of $3.75 \mathrm{~mm}^{2}$ (total slice acquisitions per run $=1920 \mathrm{scans}$ ) with anatomical reference images in the same slice locations using aT1-weighted spin-echo pulse sequence (TR = $450 \mathrm{~ms} ; \mathrm{TE}=17 \mathrm{~ms}$; interleaved; matrix $=256 \times 192 ; \mathrm{NEX}=1$; same FOV, slice thickness, and locations as the functional scans). All scans for each subject were acquired in the same session.

Prior to the application of the proposed technique, we applied preprocessing to bring homologous regions into spatial coincidence through spatial normalization. The spatial normalization of the scans to a standard template brain using the anatomical reference images was carried out in SPM99, resulting in resampling of the data to $2 \mathrm{~mm}^{3}$ isotropic voxels. The resampled data were smoothed with a Gaussian filter $\left(\right.$ FWHM $\left.15 \mathrm{~mm}^{3}\right)$. Each subject's task-related activation was analyzed individually versus the subject's rest condition, resulting in individual contrast maps giving a measurement of fMRI signal change at each voxel.

To reduce the effect of noise and sensor fluctuations in the original functional data we applied the following steps. First, we removed the effect of the background noise by subtracting the signal value measured in representative background voxels from all the voxels of the $3 \mathrm{D}$ volume. Second, we masked the data using a binary mask ex- 
tracted from the T1 anatomical atlas used as the template the data were spatially registered to. Only signal within the binary mask was included in the analysis.

\subsection{Experiments and Results}

After preprocessing (see Section 3.1) we applied the DRP algorithm to the dataset to detect discriminative activation patterns. As splitting criterion, we considered two different statistical tests: t-test and non-parametric rank-sum test. The maximum allowed tree depth was set to 3 and 4. For the significance threshold value of the stopping criterion (min correlation or maximal p-value for statistical tests) we experimented with the values of 0.05 and 0.01 . The majority of the results elucidated large hemispheric and lobar differences between Alzheimer's patients and controls for all semantic decision tasks. In particular, for CATX (semantic memory) major differences were seen in the right posterior parietal and temporal lobe regions. A more focal left inferior prefrontal region was also present. For CATF (semantic memory) group differences appeared primarily in the right frontal and distributed posterior regions including the left inferior temporal lobe. The NONPR (phonological discrimination) task showed differences in a highly dispersed set of regions including bilateral frontotemporal, parietal and subcortical sites which were more pronounced for the right than left hemisphere. Finally, in IMPREC (episodic memory), a distributed network of differences in memory associated regions including the right frontal and medial temporal regions and the left fronto-temporal neocortex was demonstrated.

The neuropathology of early AD is relatively diffuse with atrophy in widespread cortical and subcortical areas, including the medial temporal lobes and temporal parietal and frontal cortical regions [18]. On functional neuroimaging studies (fMRI and PET) patients with very early AD manifest as Mild Cognitive Impairment (MCI) often show compensatory activations outside of areas typically used by healthy elderly controls [19]. This is thought to represent the brain's recruitment of proximal and possibly distal neural units in an attempt to maintain performance in the face of progressive pathology. Therefore, the findings of multiple distributed regions that differentiate patients and controls, as detected by the DRP, may be consistent with a distributed reorganization of networks subserving the semantic memory task [11]. Due to space limitations, Figure 2 illustrates some of these regions (overlayed on the T1 atlas).

To evaluate the predictive power and association of the indicated ROIs with the disease, we proceeded with classification experiments. The goal is, given an fMRI image of a new subject, to determine the group to which it belongs, i.e., control vs. patient. For the classification model we used Neural Networks. As inputs to the classifier we used the attributes $\mathrm{V}_{\text {mean }}$ of the detected discriminative regions, standardized to have zero mean and unit standard deviation. As output we used a binary class label indicating the class of the samples. To avoid overfitting due to a small training dataset we applied one-layer perceptron networks trained by the Pocket algorithm [20]. The leave-one-out approach was employed to evaluate out of sample classification [6-7]. More specifically, the training set consisted of patients and controls with indices $1,2,3, \ldots, \mathrm{i}-1, \mathrm{i}+1, \ldots 9$ and the method was tested on patient and control with an index $\mathrm{i}$, where $i=1, \ldots, 9$. Taking into consideration the stochastic nature of the Pocket algo- 
rithm, we repeated the process of training and testing the model in each of the leaveone-out loops for 5 times and averaged the percentage of the correct predictions to obtain the reported accuracy. Table 1 shows the most characteristic classification results obtained for control and patient samples separately as well as the total classification

Table 1. Classification accuracy based on discriminative regions detected by DRP for different experimental settings and cognitive task dataset

\begin{tabular}{|c|c|c|c|c|c|c|}
\hline \multirow{2}{*}{$\begin{array}{c}\text { Cognitive } \\
\text { Test }\end{array}$} & \multirow{2}{*}{$\begin{array}{c}\text { Statistical } \\
\text { Test }\end{array}$} & \multirow{2}{*}{ Threshold } & \multirow{2}{*}{$\begin{array}{c}\text { Tree } \\
\text { Depth }\end{array}$} & \multicolumn{3}{|c|}{ Accuracy } \\
\hline & & & & Controls & Patients & Total \\
\hline$C A T X$ & t-test & 0.05 & 4 & $84.44 \%$ & $100 \%$ & $92.22 \%$ \\
\hline$C A T F$ & t-test & 0.05 & 4 & $82.22 \%$ & $97.78 \%$ & $90.00 \%$ \\
\hline IMPREC & t-test & 0.05 & 4 & $93.33 \%$ & $93.33 \%$ & $93.33 \%$ \\
\hline NONPR & t-test & 0.05 & 4 & $86.67 \%$ & $95.56 \%$ & $91.11 \%$ \\
\hline$C A T F$ & ranksum & 0.01 & 4 & $88.89 \%$ & $100 \%$ & $94.44 \%$ \\
\hline NONPR & ranksum & 0.01 & 4 & $91.11 \%$ & $100 \%$ & $95.56 \%$ \\
\hline
\end{tabular}

Table 2. Comparative classification accuracy using distributional distance-based approaches and static partitioning of the volume for the CATX set

\begin{tabular}{cccc}
\hline \multirow{2}{*}{ Alternative Method } & \multicolumn{3}{c}{ Accuracy } \\
\cline { 2 - 4 } & Controls & Patients & Total \\
\hline Maximum Likelihood - EM & 77.04 & 67.04 & 72.04 \\
Kullback-Leibler - EM & 79.26 & 57.04 & 68.15 \\
Static partitioning & $57.78 \%$ & $78.89 \%$ & $68.33 \%$ \\
\hline
\end{tabular}

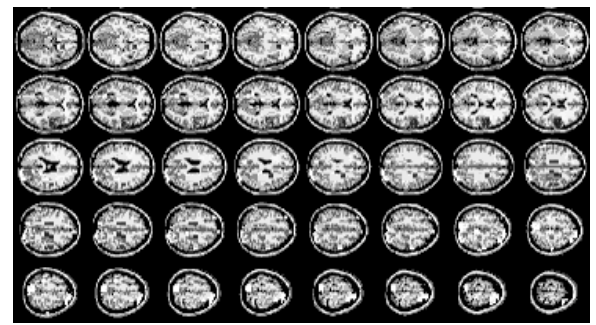

(a)

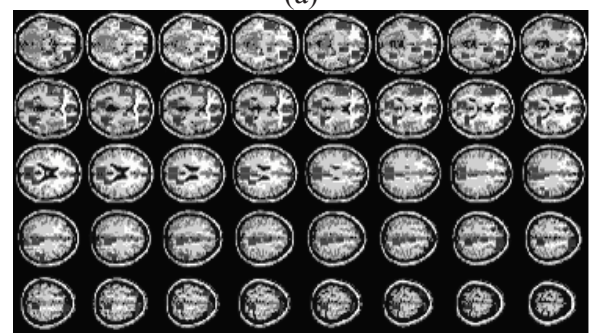

(c)

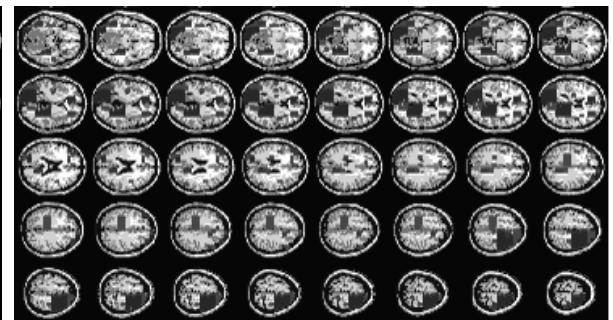

(b)

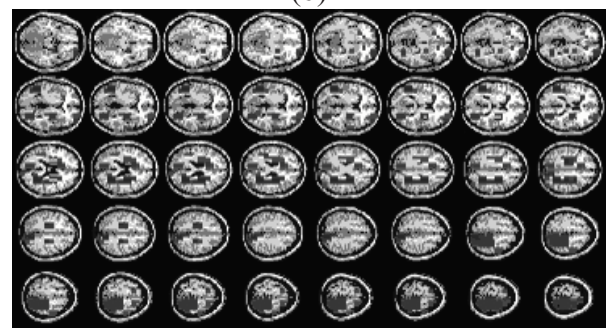

(d)

Fig. 2. The areas discovered by DRP when applied with t-test, significance threshold 0.05 and maximum tree depth 4 for (a) CATX, (b) CATF, (c) IMPREC and (d) NONPR tasks 
accuracy for each experiment; the accuracy achieved was $90 \%$ or more. As Table 2 shows, DRP outperforms other methods, such as distributional distance-based methods [4-5] and static partitioning where each dimension is split into 3 equal length bins, resulting in 27 cuboids that span the entire 3D space (best obtained results). DRP also outperformed a Fisher linear discriminant classifier approach [21]. Finally the results support the argument that the regions discovered by DRP in the specific study are indeed discriminative for $\mathrm{AD}$ and may be useful in assisting early detection of AD.

\section{Conclusions and Future Work}

We proposed and evaluated methods for the analysis of brain activation scans potentially suitable for the effective discovery of spatial activation patterns that are discriminative among different groups of subjects. The methods are applied on activation maps that are the output of SPM (operating on individual subjects independently). We replace the typical "second level" of SPM analysis (group model) by a Dynamic Recursive Partitioning (DRP) procedure that utilizes statistical tests to guide the recursive splitting of the spatial domain. We applied DRP to discover discriminative activation patterns associated with Alzheimer's disease (AD). DRP identified large hemispheric and lobar differences between Alzheimer patients and controls. It was not surprising that a broadly distributed set of sites emerged in the results. AD begins with microscopic cell loss and pathology in the medial temporal region which then spreads to broad posterior lobar areas, as reflected in the numerous reports of posterior hypometabolism seen in PET studies. Most of the sites showing classification differences are related to networks involved in human memory processes tapped by one or more of the fMRI tasks. Although there is some variation in the discovered areas related to the cognitive test that was performed to generate the datasets, the choice of the statistical test and other parameters, the most significant regions persist in all examined cases. Experiments demonstrated the ability of the indicated regions to provide efficient classification and discriminative information, improving on previous work [21] using the Fisher linear discriminant classifier. The proposed technique considers groups of voxels (spatial sub-domains) and effectively reduces the computational cost of repeated statistical tests. Experiments demonstrated that this technique outperforms other approaches, such as distributional distance-based methods, static partitioning and Fisher linear discriminant classifier. It is also more robust than methods performing voxel-wise analysis that are more prone to registration errors and variability of individual voxel values across runs, subjects and analysis techniques.

Acknowledgements. This work was supported in part by NSF (IIS-0083423, IIS0237921), NIH R01 MH68066-01A1 (funded by NIMH, NINDS, and NIA), Alzheimer's Association, Delaware State University PDF fund, and NIA AG19771. 


\section{References}

1. Koslow, S.H., Huerta, M.F., NeuroInformatics: an Overview of the Human Brain project, Mahway, NJ, Lawrence Erlbaum, (1997)

2. Megalooikonomou, V., Ford, J., Shen, L., Makedon, F., Saykin, F.: Data mining in brain imaging, Statistical Methods in Medical Research, 9 (2000) 359-394

3. Friston, KJ., Holmes, AP., Worsley, KJ., Poline, JP., Frith, CD., Frackowiak, RSJ.: Statistical parametric maps in functional imaging: a general linear approach. Human Brain Mapping (1995) 189-210

4. Lazarevic, A., Pokrajac, D., Megalooikonomou, V., Obradovic, Z.: Distinguishing Among 3-D Distributions for Brain Image Data Classification, in Proceedings of the 4th International Conference on Neural Networks and Expert Systems in Medicine and Healthcare, Milos Island, Greece (2001) 359-394

5. Pokrajac, D., Lazarevic, A., Megalooikonomou, V., Obradovic, Z.: Classification of brain image data using meaasures of distributional distance, 7th Annual Meeting of the Organization for Human Brain Mapping (OHBM01), Brighton, UK (2001)

6. Duda, R., Hart, P., Stork, D.: Pattern Classification, John Wiley and Sons, NY (2000)

7. Fukunaga, K.: Introduction to Statistical Pattern Recognition, Academic Press, San Diego (1990)

8. Coulon, O., Mangin, J.-F., Poline, J.-B., Zilbovicius, M., Roumenov, D., Samson, Y., Frouin, V., Bloch, I.: Structural group analysis of functional maps, NeuroImage, 11(6) (2000) 767-782

9. Megalooikonomou, V., Pokrajac, D., Lazarevic, A., V., Obradovic, Z.: Effective classification of 3-D image data using partitioning methods, in Proc. of the SPIE 14th Annual Symposium in Electronic Imaging: Conference on Visualization and Data Analysis San Jose, CA, Jan. (2002)

10. Megalooikonomou, V., Kontos, D., Pokrajac, D., Lazarevic, A., Obradovic, Z., Boyko, O., Saykin, A., Ford, J., Makedon, F.:Classification and Mining of Brain Image Data Using Adaptive Recursive Partitioning Methods: Application to Alzheimer Disease and Brain Activation Patterns, Human Brain Mapping Conf. (OHBM'03), New York, NY (2003) also in NeuroImage, 19 (2) S48 (2003)

11. Saykin, A.J., Flashman, L.A., Frutiger, S.A., Johnson, S.C., Mamourian, A.C., Moritz, C.H., O'Jile, J.R., Riordan, H.J., Santulli, R.B., Smith, C.A., Weaver, J.B.: Neuroanatomic substrates of semantic memory impairment in Alzheimer's disease: Patterns of functional MRI activation, Journal of the International Neuropsychological Society, 5 (1999) 377392

12. Fujimura, K. , Toriya, H., Yamaguchi, K., Kunii, T. L.: Oct-tree algorithms for solid modeling, in Computer Graphics, Theory and Applications, T. L. Kunii ed., Springer Verlag, (1983) 96-110

13. Cormen, T. H., Leadsperson, C. E., Rivest, R. L.: Introduction to Algorithms, 2nd edn., MIT Press, Cambridge (2001)

14. Devore, J.L.: Probability and Statistics for Engineering and the Sciences, 5th edn., International Thomson Publishing Company, Belmont (2000)

15. Agresti, A.: An Introduction to Categorical Data Analysis, Wiley, New York (1996)

16. Ching, J., Wong, A.: Class-dependent discretisation for inductive learning from continuous and mixed-mode data, IEEE Trans. Pattern Analysis and Machine Inteligence, 17 (1995) 641-651

17. Conover, W.J.: Practical Nonparametric Statistics, Wiley, New York (1999)

18. Flashman, L.A., Wishart, H.A., Saykin, A.J.: Boundaries Between Normal Aging and Dementia: Perspectives from Neuropsychological and Neuroimaging Investigations, in: Emory VOB and Oxman TE, editors. Dementia: Presentations, Differential Diagnosis and Nosology. Baltimore: Johns Hopkins University Press (2003) 3-30 
19. Saykin A.J., Wishart H.A.: Mild cognitive impairment: Conceptual issues and structural and functional brain correlates. Seminars in Clinical Neuropsychiatry, 8 (2003) 12-30

20. Gallant, S.I.: Perceptron-Based Learning Algorithms, in IEEE Transactions on Neural Networks, 1 (1990) 179-191

21. Ford, J., Farid, H., Makedon, F., Flashman, L.A., McAllister, T.W., Megalooikonomou, V., Saykin, A.J.: Patient Classification of fMRI Activation Maps, in Proc. of the 6th Annual International Conference on Medical Image Computing and Computer Assisted Intervention (MICCAI'03), Montreal, Canada, Lecture Notes in Computer Science 2879 (2003) 58-65 